\title{
Breast Cancer Knowledge, Perception and Breast Self- Examination Practices among Yemeni Women: an Application of the Health Belief Model
}

\author{
Khaled Abdulla Al-Sakkaf, Huda Omer Basaleem*
}

\begin{abstract}
Background: The incidence of breast cancer is rapidly increasing in Yemen with recent indications of constituting one-third of female cancers. The main problem in Yemen remains very late presentation of breast cancer, most of which should have been easily recognisable. Since stage of disease at diagnosis is the most important prognostic variable, early diagnosis is an important option to be considered for control of breast cancer in low resourced settings like Yemen. In the present study, we aimed at describing breast cancer knowledge, perceptions and breast self-examination (BSE) practices among a sample of Yemeni women. Materials and Methods: This cross-sectional study covered 400 women attending four reproductive health centres in Aden, Yemen through face-to-face interview using a structured questionnaire during April - July 2014. We collected data on sociodemographic characteristics, knowledge about breast cancer, and screening practices as well as respondents' perceptions based on the five sub scales of the Health Belief Model (HBM): perceived susceptibility; perceived severity; perceived barriers; perceived benefits; and self-efficacy. The response format was a fivepoint Likert scale. Statistical Package for Social Sciences (SPSS 20) was used for statistical analysis. Statistical significance was set at $\mathbf{p}<0.05$. Logistic regression analysis was conducted with BSE as a dependent variable. Results: The mean age of women was 26.5 ( $S . D=5.6)$ years. The majority $(89.0 \%)$ had never ever performed any screening. Two-thirds of respondents had poor knowledge. Perceived BSE benefits and self-efficacy and lower BSE barriers perception were significant independent predictors of BSE practice. Conclusions: Poor knowledge and inadequate BSE practices are prevailing in Yemen. The need for implementing culturally sensitive targeted education measures is mandatory in the effort to improve early detection and reduce the burden of breast cancer.
\end{abstract}

Keywords: Breast cancer - breast self-examination - knowledge - perception - Health Belief Model - Yemen

Asian Pac J Cancer Prev, 17 (3), 1463-1467

\section{Introduction}

Breast cancer is the most common cancer worldwide with 1.7 million women diagnosed in 2012 with breast cancer 6.3 million women alive who had been diagnosed with breast cancer in the previous five years. Breast cancer is also the most common cause of cancer death among women (522,000 deaths in 2012), and the most common type of cancer diagnosed among women in 140 of the 184 countries in the world. Now breast cancer also represents one in four of all cancer cases among women (Ferlay et al., 2013). It is estimated that one out of every eight women (1 in 8) live beyond the age of seventy in Western countries are likely to suffer from breast cancer (ACS, 2005). Breast cancer will remain an important issue for public health in developed and the developing countries because of the rapidly rising number of new cases in developing countries and the high prevalence globally (Rastgoi, 2004). Since the 2008 estimates, breast cancer incidence has increased by more than $20 \%$, while mortality has increased by $14 \%$ (Bray et al., 2013).

In Yemen, breast cancer is the first of the five most common reported cancers among Yemeni people (16.8\%), as well as among Yemeni women (31.6\%) (Ba Saleem et al., 2013), and its incidence rate among Yemeni women is dramatically growing during the last years (Ba Saleem et al., 2010; El-Zaemey et al., 2012). It is also clear from cancer incidence reports that issued from Aden cancer center for research and registry (Harhra and $\mathrm{Ba}$ Saleem, 2012; Ba Saleem, 2013), Hadramout cancer center (Badheeb, 2012) and the national oncology center registry in Sanaa (El-Zaemey et al., 2012) that Yemeni breast cancer patients like others of breast cancer patients in the Arab countries (El Saghir, 2007) presents almost 10 years younger than in USA and Europe. Median age at presentation is $48-52$ and $50 \%$ of all cases are below the age of 50 whereas only $25 \%$ of cases in industrialized nations are below the age of 50 years (El Saghir, 2007; 
Saadat, 2008).

The three screening methods recommended for breast cancer includes BSE, clinical breast examination (CBE), and mammography. Unlike CBE and mammography, which require hospital visit and specialized equipments and expertise, BSE is inexpensive and is carried out by women themselves (Humphrey et al., 2002; Saslow et al., 2007; Ba'amer, 2010). BSE is an important choice for screening and an appropriate choice to reduce the incidence and mortality of breast in developing countries including Yemen. Therefore, increasing the awareness and knowledge of women as well as increasing the behavior of BSE will decrease the stage of the disease upon presentation and improve the quality of life and survival which relate directly to the stage of disease (Parkin et al., 2005, Smith, 2005). Accordingly, good knowledge, perception, and practices of the community is necessary for a screening program particularly BSE. Therefore, this study is conducted to identify the knowledge, perception and screening practices of Yemeni women regarding breast cancer and BSE practices using the HBM as a psychosocial model that accounts for health behaviors by identifying factors associated with individuals' beliefs which influence their behaviors (Champion, 1984).

\section{Materials and Methods}

This is a cross sectional study conducted in Aden city from April to July 2014 among 400 women attending four primary health care centres.

\section{Study area and population}

The study was carried out in Aden city, which is the economic capital of Yemen. Aden has eight districts with 18 health centres in which primary health services such as reproductive, immunization, and child development assessment are offered to healthy women and their children. The study population was women attending health centres in Aden city seeking for the preventive and curative health services provided in these centres.

\section{Sampling}

The required sample of studied women was calculated assuming that the expected proportion of the breast cancer knowledge among women in their reproductive age is 50\% with absolute precision of 5\%. The needed sample size was estimated (using Lwanga and Lemeshow 1991 practical manual) to be 384 which was approximated to reach 400 women from those attending the health centres as a total sample size. Women who were previously diagnosed with breast cancer, who were pregnant or who was breast feeding at the time of recruitment were excluded from the study.

To obtain this sample, four primary health care centres from the total 18 centres were selected by simple random sampling and then 100 women were selected from each chosen centre. After the selection of the first woman who attend the centre and she was eligible and agreed to take part in the study, then by simple systematic sampling technique, the second prospective subject was the fifth women attending the health centre if she was eligible and if she consented to participate in the study. If this subject did not meet the sampling criteria or if she refused to participate, then the next $\left(6^{\text {th }}\right)$ was selected and so on. Thus every other five women were recruited until 100 subjects were attained from each of the four selected health centres.

\section{Measures}

A face-to-face interview structured questionnaire was used as data collection instrument for this study. BSE was the dependent variable in this study. In addition to the sociodemographic characteristic (age, level of education, and marital status), history of breast problems, women were asked two questions about performing BSE: (1) Do you perform breast examination? (2) If yes, how often do you perform it? BSE was considered "regular" if it had been performed at least once a month and irregular if it had not been monthly. Breast cancer and breast cancer screening related knowledge were assessed through 17 statements. Correct answer for each statement was assigned one mark whereas incorrect answer and non response given zero, so the total maximum score for knowledge was 17 marks. Then the respondents' responses were divided according to their answers into three levels as follows: 0-6 marks=low level of knowledge; 7-11 marks=intermediate level of knowledge; 12-17 marks=high level of knowledge.

Using the Champion's revised Health Belief Model Scales (CHBMS) (Champion and Scott, 1997), HBM variables including susceptibility, seriousness, perceived benefits, perceived barriers, self-efficacy and health motivation associated with breast cancer screening were measured. All CHBMS sub-scales except health motivation were adapted for the present study. The CHBMS sub-scales in the questionnaire measured through 31 items. All items prepared in a Likert format offered five response choices ranging from "strongly disagree (scores 1 point)" to "strongly agree (scores 5 points)". Higher scores indicated a positive attitude towards BSE except for barriers to BSE as follows: $i$ ). Susceptibility of breast cancer (three items) scored from 3 to 15; ii). Seriousness of breast cancer (six items) scored from 6 to 30; iii). BSE benefits (four items) scored from 4 to 20; $v i$ ). BSE barriers (eight items) scored from 8 to 40; $v$ ). BSE self-efficacy (10 items) scored from 10 to 50

The revised CHBMS was translated to Arabic language using a backward-forward translation technique. To do this, the Arabic translation was validated by professional judges of panel of experts who translated the CHBMS items from English to Arabic language and then it was back-translated into English. Minor translation adjustments were carried out until the two versions (Arabic /English formats) were identical. Thereafter, Cronbach's alpha reliability coefficients was measured and found to be ranged from 0.71 to 0.92 for the subscales.

\section{Data analysis}

Data were analyzed by using SPSS (version 20.0) software. Categorical variables were described by using frequency distribution and percentage. Then, for analyzing, sample was divided into two parts according to whether or not they had performed BSE at all. Chi square test was used to measure the differences between 
Breast Cancer Knowledge, Perception and Breast Self-examination Practices among Yemeni Women

categorical variables and the independent t-tests was used to compare health belief model constructs between women who performed BSE and who did not perform it. To evaluate the predictive value of the variables of BSE performance, a logistic regression was performed with BSE performance as dependent variable, and health belief model constructs (perceived susceptibility, perceived seriousness, perceived benefits, and perceived barrier, self-efficacy) as predictor variables. $\mathrm{P}$ value $<0.05$ was considered statistically significant.

\section{Ethical considerations}

Permission was obtained from Research Ethics Committee of the Faculty of Medicine and Health Sciences before initiating the research. Before any data were obtained, women were informed about the aim of

Table 1. The Characteristics of the Respondents and their Level of Knowledge about Breast Cancer and Breast Cancer Screening

\begin{tabular}{lcc}
\hline Variable & No & $\%$ \\
\hline Age & & \\
$\quad$ Mean (Sd) & 26.5 (S.D $=5.6)$ \\
Marital status & & \\
$\quad$ Married & 346 & 86.5 \\
$\quad$ Currently unmarried & 54 & 13.5 \\
Educational level & & \\
Illiterate & 35 & 8.7 \\
Read \& write & 46 & 11.5 \\
$\quad$ Primary & 102 & 25.5 \\
Secondary & 139 & 34.8 \\
University & 78 & 19.5 \\
History of breast problems & & \\
$\quad$ Yes & 42 & 10.9 \\
$\quad$ No & 358 & 89.1 \\
Performing BSE & & \\
$\quad$ Yes & 44 & 11 \\
No & 356 & 89 \\
Breast cancer screening related knowledge & \\
Low level of knowledge & 270 & 67.5 \\
Intermediate level of knowledge & 92 & 23 \\
High level of knowledge & 38 & 9.5 \\
\hline
\end{tabular}

the study and oral consent was obtained from each of them. To ensure confidentiality, women were asked not to provide their names.

\section{Results}

Background characteristics of the study population

The study involved 400 women attending the health centres in Aden city. The mean age of the participants was 26.5 ( $\mathrm{S} . \mathrm{D}=5.6)$. The majority of studied women $(86.5 \%)$ were married. Around one fifth $(19.5 \%)$ of the participants were of university level of education and the highest percentage of them $(34.8 \%)$ was of secondary level of education. Only about one tenth of the women (10.9\%) had a history of breast problems. Just around one tenth of the women (11.0\% or 44$)$ had ever performed BSE during their life and only $4.5 \%$ (18 of 400) performed it at least monthly. The study also revealed that two thirds $(67.5 \%)$ of the participants had low level of knowledge and the remaining one third were categorized as intermediate $(23.0 \%)$ and high (9.5\%) levels of knowledge (Table 1).

Comparing women who performed BSE $(n=44)$, and who did not perform it $(n=356)$, there were no statistical differences in BSE performance with respect to age, marital status, level of education, history of breast problems, or the level of knowledge ( $\mathrm{p}>0.05)$.

Factors arising from the HBM were compared for BSE performers and non-performers (never done) in Table 2. As the data in this table indicate, perceived BSE benefits and self-efficacy among women who performed BSE were significantly higher than among those who never performed it $(\mathrm{p}<0.05)$. On the other hand, perceived BSE barriers among BSE performers were significantly lower than those of non-performers $(\mathrm{p}<0.05)$.

Table 3 shows that women who perceived fewer BSE barriers (OR=0.63) and more BSE self-efficacy ( $\mathrm{OR}=1.13)$ were more likely to perform BSE.

\section{Discussion}

Breast cancer incidence is increasing year by year.

Table 2. Comparison of Health Belief Model factors among performers and non-performers of BSE

\begin{tabular}{lccccc}
\hline Sub-scale & Performing BSE & \multicolumn{2}{c}{ Score Range } & & \\
\cline { 2 - 5 } & & $\begin{array}{c}\text { Yes (n=44) } \\
\text { Mean (SD) }\end{array}$ & $\begin{array}{c}\text { No (n=356) } \\
\text { Mean (SD) }\end{array}$ & $\mathrm{t}$ & $\mathrm{p}^{*}$ \\
\hline Susceptibility & $3-15$ & $7.2(2.3)$ & $6.9(2.1)$ & 0.89 & 0.38 \\
Seriousness & $6-30$ & $19.8(4.9)$ & $20.2(4.8)$ & 0.52 & 0.6 \\
BSE benefits & $4-20$ & $17.4(3.1)$ & $16.3(3.5)$ & 2.04 & 0.02 \\
BSE barriers & $8-40$ & $18.2(4.8)$ & $21.7(5.3)$ & 4.17 & 0.0001 \\
BSE self - efficacy & $10-50$ & $37.2(7.1)$ & $28.4(6.8)$ & 8.06 & 0.0001 \\
\hline
\end{tabular}

Table 3. Logistic Regression Analysis of Health Belief Model Factors for Predicting BSE Performance

\begin{tabular}{lccccc}
\hline HBM construct & Regression coefficient B & $\mathrm{SE}( \pm)$ & OR & $95 \%$ CI & p-value \\
\hline Susceptibility & $3-15$ & $7.2(2.3)$ & 1.02 & $0.88-1.17$ & 0.96 \\
Seriousness & $6-30$ & $19.8(4.9)$ & 1.05 & $0.97-1.11$ & 0.17 \\
BSE benefits & $4-20$ & $17.5(3.1)$ & 0.89 & $0.77-1.05$ & 0.06 \\
BSE barriers & $8-40$ & $18.2(4.8)$ & 0.63 & $0.59-0.72$ & $<0.001$ \\
BSE self - efficacy & Oct-50 & $37.2(7.1)$ & 1.13 & $1.06-1.18$ & 0.002 \\
Constant & & & & \\
\hline
\end{tabular}


Early detection is still the first priority for cancer control program. There is no doubt that screening methods recommended for breast cancer including BSE, can be efficient in reduction breast cancer mortality (Bray $\mathrm{F}$ et al., 2013).

Breast cancer is one of the most frequent cancers occurring among Yemeni women characterized by delay in diagnosis and treatment as well as the occurrence in younger group (El-Zaemey et al., 2012; Harhra and Ba Saleem, 2012). Therefore, BSE will provide a reasonable screening method for early detection of treatable breast cancers and Yemeni women need to exercise some sense of control over breast cancer, especially given the limited availability of mammograms and clinical breast examinations compared to developed countries. Thus, an appropriate knowledge and attitude of the Yemeni community about breast cancer screening programs may be the most important necessity for empowering Yemeni women and encourage them to conduct BSE.

Our study revealed that only few participants (4.5\%) performed BES on a regular basis. Consistent with our study, regular BES examination were performed only by $4.0 \%$ in Saudia Arabia (Yousuf et al., 2012), 4.2\% of Yemeni university students (Ba'amer, 2010) and 7.1\% in Iran (Tavafian et al.,2009). In contrast to our results, higher rates of regular BES performance among Turkish $(30.7 \%)$ and Iranian women (12.9\%) as well as among Iranian female health workers (14.9\%) were reported by (Guvenc et al., 2012), (Nafissi et al., 2012) and (Shiryadi et al., 2014) respectively.

According to our study, $11.0 \%$ of our respondents performed BSE occasionally and a similar figure (13.8\%) was reported by Akhtari-Zavare et al (2014) study in Iran. In contrast to our findings, higher rates of BSE performance by women were reported in Saudi Arabia by Abolfotouh et al (2015) and in Iran by Nafissi et al (2012).

The majority of the respondent women in this study had low level of knowledge $(67.5 \%)$, which is slightly higher than the percentage of university female students $(58.6 \%)$ that had low level of knowledge reported by Ba'amer in Al-Mukalla city - Yemen, 2010. In contrast with this result in Yemeni women, report from Morocco and Saudi Arabia showed that higher percentage of Moroccans nurses (Ghanem et al, 2011) and primary health care nurses in Jeddah city (Yousuf et al, 2012) had have high level of knowledge about breast cancer. The low level of knowledge found in this study is keeping also with reports of other researchers from Malaysia (Subramanian et al, 2013) and Iran (Tazhibi and Feizi, 2014).

This study showed that there is no relationship between the low rate of Yemeni women performance of BSE and their socio-demographic characteristics (age, marital status and educational level), their previous history of breast problems and their breast cancer and breast cancer screening related knowledge. Similar to our results, a study of Malaysian teachers identified that there was no association between socio demographic characteristics and family history of breast cancer and BSE practice (Parsa et al., 2008). On the other hand, there are a number of studies has shown that women low rate of BSE performance was related to socio-economic status, educational level and breast cancer related knowledge and BSE practices (Montazeri et al., 2003; Yavari and Pourhoseingholi, 2007; Montazeri et al., 2008).

In contrary to the constructs of HBM that predicts women who perceive themselves to be susceptible to breast cancer (perceived susceptibility) and who also believe that breast cancer is a serious disease (perceived seriousness) are more likely to practice regular BSE, the present study did not establish any relations between perceived susceptibility/seriousness and BSE. One explanation of this finding may be related to the low level of knowledge of Yemeni women regarding breast cancer.

Similar to our results from univariate analysis, Tavafian et al (2009), showed that Iranian women who regularly performed $\mathrm{BSE}$, perceived more BSE benefits, fewer BSE barriers and more BSE self-efficacy than those women who had never performed BSE, while the findings of logistic regression analysis indicated that perceived barriers and self-efficacy were the only health belief variables to predict BSE performance. This study results are parallel to those of Tavafian et al study (2009).

In conclusion, Poor knowledge and inadequate BSE practices are prevailing among the respondent' women living in the Aden, Yemen. These results indicate the urgent need to implement targeted, continuous, concentrated and mandatory awareness-raising measures in the community, taking into account the social and cultural background to improve early detection and reduce the burden of breast cancer in Yemen. These mandatory efforts should focus on encouraging women to practice BSE.

Furthermore, the findings of this study indicated that only perceived BSE benefits and self-efficacy and perceived BSE barriers of the HBM constructs were associated with the practice of BSE among this sample of Yemeni women. Therefore, these and other mandatory efforts to improve awareness of Yemeni women's perceived susceptibility and perceived seriousness of the breast cancer, the benefits and self-efficacy of BSE practices as well as to reduce the barriers of BSE practices are strongly recommended.

\section{References}

Abolfotouh1 MA, BaniMustafa AA, Mahfouz AA, et al (2015). Using the health belief model to predict breast self examination among Saudi women. BMC Public Health, 15, 1163-74.

Akhtari-Zavare M, Ghanbari-Baghestan A, Latiff LA, et al (2014). Knowledge of breast cancer and breast selfexamination practice among Iranian women in Hamedan, Iran. Asian Pac J Cancer Prev, 15, 6531-4.

American Cancer Society (ACS) (2005). Breast Cancer Facts and Figures 2005-2006. Atlanta, Ga: American Cancer Society.

Ba'amer AA (2010). Awareness and practice of breast cancer and breast-self examination among university students in Yemen. Asian Pac J Cancer Prev, 11, 101-5.

Ba Saleem HO, Bawazir AA, Moore M, Al-Sakkaf KA (2010). Five years cancer incidence in Aden Cancer Registry, Yemen (2002-2006). Asian Pac J Cancer Prev, 11, 507-11.

Ba Saleem HO (2013). Cancer incidence report 2007-2011. Aden Cancer Registry and Research Centre, Aden. Yemen.

Badheeb AM, Ba'amer AA (2012). Cancer incidence report 2000-2011. hadhramout cancer registry, hadhramout cancer 
foundation. yemen.

Bray F, Ren JS, Masuyer E, et al (2013). Global estimates of cancer prevalence for 27 sites in the adult population in 2008 . Int J Cancer, 132, 1133-45.

Champion VL(1984). Instrument development for health belief model constructs. Adv Nurs Sci, 6, 73-85.

Champion VL, Scott CR (1997). Reliability and validity of breast cancer screening belief scales in African American women. Nurs Res, 46, 331-7.

El-Zaemey S, Nagi N, Fritschi L, et al (2012). Breast cancer among Yemeni women using the National Oncology Centre Registry 2004-2010. Cancer Epidemiol, 36, 249-53.

El Saghir NS, Khalil MK, Eid T, et al (2007). Trends in epidemiology and management of breast cancer in developing Arab countries: a literature and registry analysis. Int J Surg, 5, 225-33.

Ferlay J, Soerjomataram I, Ervik M, et al (2013). GLOBOCAN 2012, cancer incidence and mortality worldwide: IARC cancer base No. 11 [Internet]. lyon, france: international agency for research on cancer.

Ghanem S, Glaoui M, Elkhoyaali S, et al (2011). Knowledge of risk factors, beliefs and practices of female healthcare professionals towards breast cancer, Morocco. Pan Afr Med J, 10, 21.

Guvenc I, Guvenc G, Tastan S, et al (2012). Identifying women's knowledge about risk factors of breast cancer and reasons for having mammography. Asian Pac J Cancer Prev, 13, 4191-7.

Harhra NA, BaSaleem HO (2013). Trends of breast cancer and its management in the last twenty years in aden and adjacent governorates, yemen. Asian Pac J Cancer Prev, 13, 4347-51.

Heidari Z, Mahmoudzadeh-Sagheb HR, Sakhavar N (2008). Breast cancer screening knowledge and practice among women in southeast of Iran. Acta Med Iranica, 46, 321-8.

Humphrey L, Helfanol M, Chan P, et al (2002). Breast cancer screening: a summary of the evidence for the US preventive services task force. Ann Inter Med, 137, 347-60.

Lwanga, SK, Lemshow, S (1991). Sample size determination in health studies; a practical manual. geneva, World Health Organisation.

Montazeri A, Haji-Mahmoodi M, Jarvandi S (2003). Breast self-examination: Knowledge, attitudes and practices among female health care workers in Tehran, Iran.J Public Health Med, 25, 154-5.

Montazeri A, Vahdaninia M, Harirchi I, et al (2008). Breast cancer in Iran: need for greater women awareness of warning signs and effective screening methods. Asia Pac Fam Med, 7, 6-12.

Nafissi N, Saghafinia M, Motamedi MK, et al (2012). A survey of breast cancer knowledge and attitude in Iranian women. J Can Res Ther, 8, 46-9.

Parkin DM, Bray F, Ferlay J, et al (2005). Global cancer statistics, 2002. CA Cancer J Clin, 55, 74-108.

Parsa P, Kandiah M, Zulkefli MNA, et al (2008). Knowledge and behaviour regarding breast cancer screening among female teachers in Selangor, Malaysia. Asian Pac J Cancer Prev, 9, 221-7.

Rastgoi M (2004). Increase in cancer death by the year 2020 . geneva: world health organization.

Saslow D, Boetes C, Burke W, et al (2007). American Cancer Society guidelines for breast screening with MRI as an adjunct to mammography. CA Cancer J Clin, 57, 75-89.

Saadat S (2008). Can we prevent breast cancer? Int J Health Sci (Qassim), 2, 167-70.

Shiryazdi1 SM, Kholasehzadeh G, Neamatzadeh H, et al (2014). Health beliefs and breast cancer screening behaviors among Iranian female health workers. Asian Pac J Cancer Prev, 15, 9817-22.
Smith RA, Cokkinides V, Eyre HJ (2005). American Cancer Society guidelines for the early detection of cancer. $C A$ Cancer J Clin, 55, 31-44.

Subramanian P, Oranye NO, Masri AM, et al (2013). Breast cancer knowledge and screening behaviour among women with a positive family history: A cross sectional study. Asian Pac J Cancer Prev, 14, 6783-90.

Tavafian S, Hasani L, Aghamolaei T, et al (2009). Prediction of breast self-examination in a sample of Iranian women: an application of the health belief model. BMC Women's Health, 9, 37-43.

Tazhibi M, Feizi A (2014). Awareness levels about breast cancer risk factors, early warning signs, screening and therapeutic approaches among iranian adult women: a large population based study using latent class analysis. BioMed Res Int, 306352- 61.

Yavari P, Pourhoseingholi MA (2007). Socioeconomic factors association with knowledge and practice of breast selfexamination among Iranian women. Asian Pac J Cancer Prev, 8, 618-22.

Yousuf SA, Al Amoudi SM, Nicolas W, et al (2012). Do Saudi nurses in primary health care centres have breast cancer knowledge to promote breast cancer awareness? Asian Pac J Cancer Prev, 13, 4459-64. 